\title{
Small-Molecule Ice Recrystallization Inhibitors Improve the Post- Thaw Function of Hematopoietic Stem and Progenitor Cells
}

\author{
Jennie G. Briard, ${ }^{\dagger}$ Suria Jahan, ${ }^{\ddagger}$ Priya Chandran, ${ }^{\S}$ David Allan, ${ }^{\S}$ Nicolas Pineault, ${ }^{\ddagger}$ and Robert N. Ben*, ${ }^{\dagger}$ \\ ${ }^{\dagger}$ Department of Chemistry and Biomolecular Sciences, University of Ottawa, 10 Marie Curie, Ottawa, Ontario K1N 6N5, Canada \\ ${ }^{\ddagger}$ Center for Innovation, Canadian Blood Services, 1800 Alta Vista Drive, Ottawa, Ontario K1G 4J5, Canada \\ ${ }^{\S}$ Faculty of Medicine, University of Ottawa, 451 Smyth Road, Ottawa, Ontario K1H 8M5, Canada
}

Supporting Information

ABSTRACT: The success of hematopoietic stem cell transplantation depends in part on the number and the quality of cells transplanted. Cryoinjuries during freezing and thawing reduce the ability of hematopoietic stem and progenitor cells (HSPCs) to proliferate and differentiate after thawing. Up to $20 \%$ of the patients undergoing umbilical cord blood (UCB) transplant experience delayed or failed engraftment, likely because of the inadequate hematopoietic potency of the unit. Therefore, the optimization of cryopreservation protocols, with an emphasis on the preservation of HSPCs, is an important issue. Current protocols typically utilize a $10 \%$ dimethyl sulfoxide cryoprotectant solution. This solution ensures $70-80 \%$ post-thaw cell viability by diluting intra-
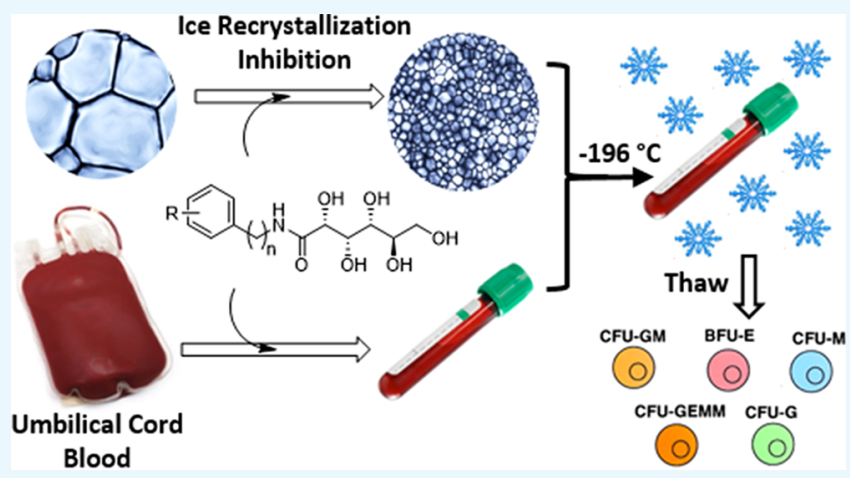
cellular solutes and maintaining the cell volume during cryopreservation. However, this solution fails to fully protect HSPCs, resulting in the loss of potency. Therefore, a new class of cryoprotectants ( $N$-aryl-D-aldonamides) was designed and assessed for the ability to inhibit ice recrystallization and to protect HSPCs against cryoinjury. Several highly active ice recrystallization inhibitors were discovered. When used as additives to the conventional cryoprotectant solution, these nontoxic small molecules improved the preservation of functionally divergent hematopoietic progenitors in the colony-forming unit and long-term cultureinitiating cell assays. By contrast, structurally similar compounds that did not inhibit ice recrystallization failed to improve the post-thaw recovery of myeloid progenitors. Together, these results demonstrate that the supplementation of cryopreservation solution with compounds capable of controlling ice recrystallization increases the post-thaw function and potency of HSPCs in UCB. This increase may translate into reduced risk of engraftment failure and allow for greater use of cryopreserved cord blood units.

\section{INTRODUCTION}

Transplantation of hematopoietic stem and progenitor cell (HSPC) products offers life-saving treatment for patients suffering from hematological cancers and nonmalignant hematological diseases such as hemoglobinopathies, immunodeficiency syndromes, and inherited metabolic disorders. ${ }^{1,2}$ Moreover, HSPCs are increasingly used for novel and emerging regenerative therapies, including the treatment of neurological disorders, cardiac disease, and diabetes. ${ }^{3}$ In comparison to bone marrow (BM) or mobilized peripheral blood, hematopoietic stem cell transplantation using umbilical cord blood (UCB) can be associated with delayed neutrophil and platelet engraftment due to the limited volume that can be collected and the resulting reduced dosage of cells. ${ }^{4}$ However, UCB offers several advantages such as ready availability, reduced risk of graftversus-host disease (GVHD), negligible risk to donor, and lessstringent human leukocyte antigen (HLA)-matching requirements. 5,6

Although stem cell grafts from adult donors are collected and infused as quickly as possible without cryopreservation, UCB units are processed and stored at $-196{ }^{\circ} \mathrm{C}$ for future use. Moreover, in the case of autologous hematopoietic transplantation, patients' cells are collected, cryopreserved, and reinfused following the conditioning treatment to rescue hematopoiesis. ${ }^{7}$ Cryopreservation introduces several issues that may compromise the success of transplantation. First, HSPCs are cryopreserved using the cryoprotectant dimethyl sulfoxide (DMSO). ${ }^{8}$ The DMSO present in the thawed HSPC product has been shown to have adverse effects on the gastrointestinal, renal, hepatic, central nervous, cardiovascular, and respiratory systems. ${ }^{7,9-12}$ In an effort to reduce toxicity, the removal of DMSO before transfusion has been employed for patients at risk with pre-existing conditions. ${ }^{13-15}$ However, Yang et al. have demonstrated that removing DMSO through repeated washing decreases viability and recovery of UCB CD34+ cells. ${ }^{16}$ The second issue with cryopreservation is that

Received: August 9, 2016

Accepted: October 21, 2016

Published: November 28, 2016 
high levels of apoptotic CD34+ cells (up to 30\%) are observed following cryopreservation, and this is correlated with a reduced ability to engraft. ${ }^{16-19}$ Additionally, Sasnoor et al. have found that cryopreservation impairs growth factor responsiveness, and this is correlated with a reduced ability to proliferate and differentiate post-thaw. ${ }^{20-22}$

Recently, Ben et al. reported that the supplementation of the cryoprotectant solution with mono- and disaccharides possessing the ability to inhibit ice recrystallization improved the postthaw viability of $\mathrm{CD} 34+$ cells from cryopreserved $\mathrm{UCB}^{23}$ However, the success of HSPC transplantation is directly correlated with both the number and the quality of cells transplanted. ${ }^{24-31}$ A recent study correlated prefreeze and postthaw cell characteristics to determine the best indicators for successful engraftment and concluded that the colony-forming unit (CFU) dose was the best indicator for successful engraftment. ${ }^{32}$ Therefore, we sought to assess the post-thaw clonogenic potential of HSPCs cryopreserved with small molecules capable of controlling ice growth or recrystallization. In this report, a new class of novel small molecules, $N$-aryl-Daldonamides (Figure 1), was rationally designed and assessed

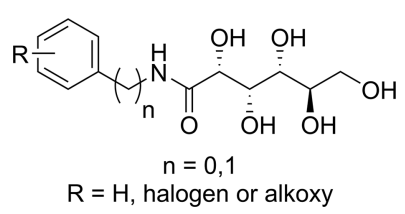

Figure 1. General structure of $N$-aryl-D-aldonamides.

for their ability to inhibit ice recrystallization. Several very effective ice recrystallization inhibitors (IRIs) were discovered. When used as additives to the conventional 10\% DMSO cryoprotectant solution, these nontoxic small molecules improved the post-thaw recovery from hematopoietic progenitors from cryopreserved UCB units.

\section{RESULTS AND DISCUSSION}

2.1. Ice Recrystallization Inhibition Activity of $\mathbf{N}$-ArylD-aldonamides. A series of $N$-aryl-D-aldonamides were synthesized and assessed for their ability to inhibit ice recrystallization using the splat-cooling assay (Figure 2). Briefly, compounds were dissolved at $22 \mathrm{mM}$ in phosphate buffered saline (PBS), except for compound 2, which was insoluble at 22 $\mathrm{mM}$ and assessed at $11 \mathrm{mM}$. A $10 \mu \mathrm{L}$ droplet of this solution was dropped onto a precooled $\left(-80{ }^{\circ} \mathrm{C}\right)$ block of polished aluminum to form a frozen wafer, which was then held at -6.4 ${ }^{\circ} \mathrm{C}$ for $30 \mathrm{~min}$. The wafer was then photographed, and the ice crystal sizes were calculated using ImageJ analysis software (see Supporting Information for details). The inhibitory activity is represented as a percentage of mean grain size (MGS) relative to a PBS positive control for ice recrystallization. As such, a smaller percentage represents a more highly active molecule (i.e., smaller ice crystals in the sample).

Compound 1 was moderately active with a MGS of $65 \%$ when compared with the PBS control. However, the addition of a fluorine atom at position 2 on the aromatic ring (compound 2) resulted in a highly active IRI with a MGS of $3 \%$ (Figure 2 ). Interestingly, a fluorine substituent in the para-position (3) abolished much of this activity (MGS of 90\%), whereas a chlorine substituent in the para-position (4) partially restored the lost IRI activity. Conversely, when this chlorine substituent was positioned in the ortho-position (5), the compound was inactive. Interestingly, the introduction of a methoxy functional group in the para-position (6) resulted in a very effective IRI with a MGS of $4 \%$, whereas substitution with the same functional group in the ortho-position (7) abolished this activity (Figure 2). Compound 8 with a carbon linker between the aryl ring and the amide bond possessed an IRI activity of approximately $40 \%$. Compound 9, having two fluorine substituents in positions 2 and 6 of the aromatic ring, was a highly IRI active molecule with a MGS of $13 \%$. However, when the fluorine substituents were in positions 3 and 5 of the aromatic ring (10), the molecule was inactive (Figure 2). In summary, these results show that IRI molecules $\mathbf{2}$ and $\mathbf{6}$ are the most potent IRI compounds identified in this directed screen followed by 4 and 9 . Conversely, structurally similar compounds 3, 5, 7, and 10 were inactive IRIs that were utilized as controls for the IRI active molecules.

2.2. Impact of IRIs on the Post-thaw Viability of UCB CD34+ Cells from Leukocyte Concentrates. In the clinic, leukocyte concentrates (LCs) (i.e., buffy coat) consisting of total nucleated cells (TNCs) containing myeloid and lymphoid cells and HSPCs are isolated from units by red blood cell (RBC) depletion using hydroxyethyl starch. UCB LCs were extracted from the UCB, and the total number of viable CD34+ cells was quantified by flow cytometry using the single-platform ISHAGE method for CD34 cell enumeration. Small-scale cryopreservation experiments were designed to carefully mimic clinical preservation protocols (DMSO and dextran in $0.9 \%$

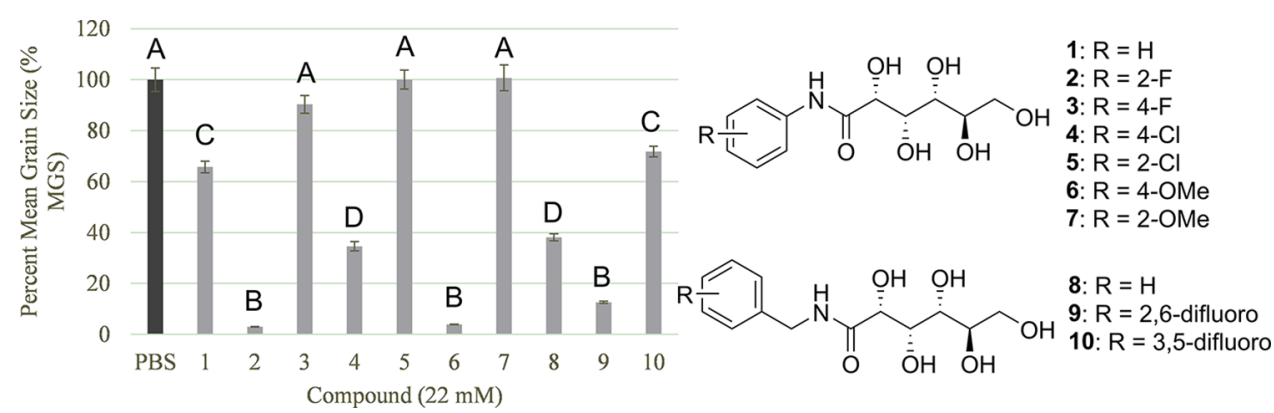

Figure 2. Structures and IRI activities of $N$-aryl-D-aldonamides 1-10. Compounds were assessed at $22 \mathrm{mM}$ except for compound 2, which was insoluble at $22 \mathrm{mM}$ and tested at $11 \mathrm{mM}$. IRI activity is expressed as a percentage of MGS relative to a PBS positive control for ice recrystallization. Error bars are reported as the standard error of the mean (SEM). Statistical significance $(p<0.05)$ was assessed using a one-way analysis of variance (ANOVA) with a Tukey multiple comparison test. Compounds with similar activities $(p>0.05)$ are marked with the same letter above the bars in the graph, and statistical differences $(p<0.05)$ are indicated by the use of different letters above the bars. 


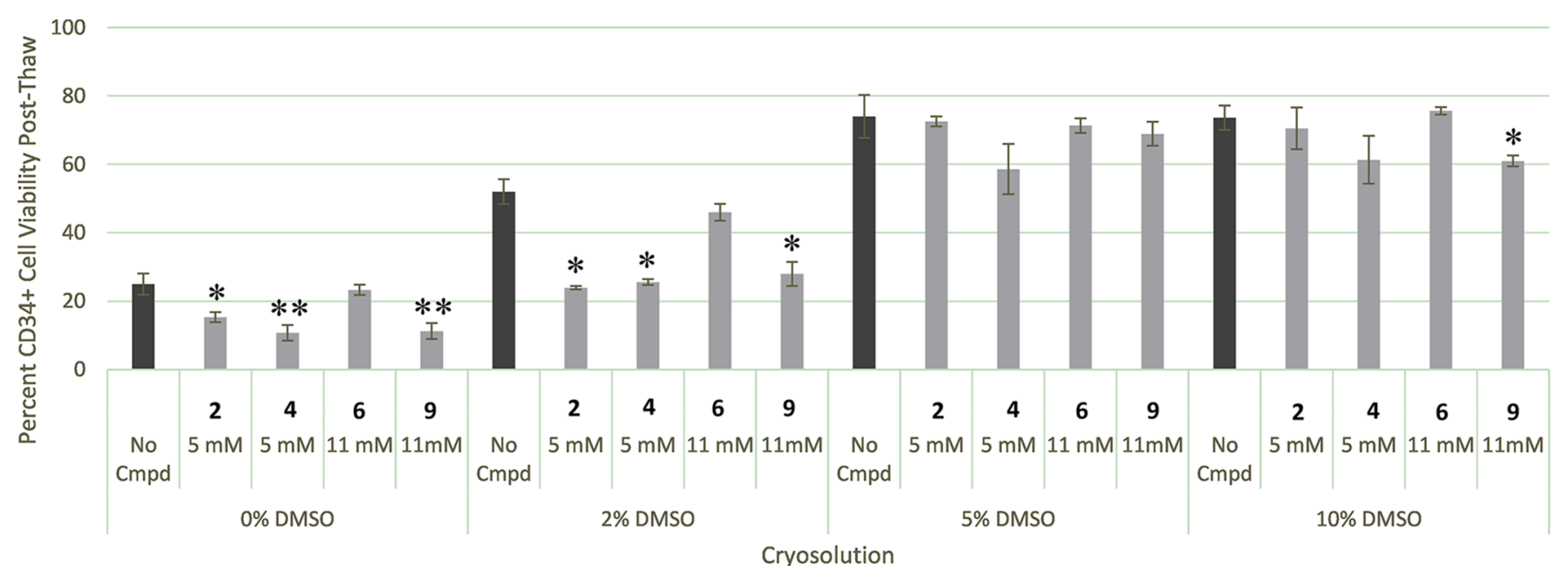

Figure 3. Post-thaw viability of CD34+ cells in LCs cryopreserved in various concentrations of DMSO and IRI active compounds $\mathbf{2}$, 4, 6, and 9 . Mean \pm SEM presented of 3-7 independent experiments. Viability was assessed by flow cytometry using the ISHAGE-gating strategy with 7-AAD. Statistical significance, marked by asterisks, was assessed using ANOVA with a Dunnett's test for comparison to the control (no compound) with a $95 \%(*)$ or $99 \%(* *)$ confidence level.

saline) to effectively compare the cryopreservation success of HSPCs that are cryopreserved with IRI active molecules.

We hypothesized that by supplementing this cryoprotectant solution with IRIs, the concentration of DMSO could be reduced without influencing post-thaw cell viability. Therefore, the LCs were cryopreserved with final DMSO concentrations of $0,2,5$, and $10 \%$ DMSO (using a 1:5 dilution with the LC). These cryoprotectant solutions were also supplemented with IRI active compounds 2, 4, 6, and 9. The cryoprotectant solution was added to LCs $(50000 \mathrm{CD} 34+$ cells $/ \mathrm{mL})$ and then cooled at a rate of $1{ }^{\circ} \mathrm{C} / \mathrm{min}$ to $-80{ }^{\circ} \mathrm{C}$ before storage in a liquid nitrogen Dewar flask for $48 \mathrm{~h}$. The samples were then thawed in a $37{ }^{\circ} \mathrm{C}$ water bath and diluted, and the post-thaw viability was assessed by flow cytometry using 7-AAD. As shown in Figure 3, there is no increase in the CD34+ cell viability upon supplementation of the 0 and $2 \%$ DMSO cryoprotectant solution with IRIs. In fact, in most instances, there are statistically significant decreases in the post-thaw viability. When 5\% DMSO is used, there is no difference in the post-thaw viability with the IRIs. The same results were obtained with $10 \%$ DMSO with the exception of a slight reduction in the viability with 9 . These results suggested a cytotoxic effect by IRIs. However, when the IRI compounds were assessed for cytotoxicity using the MTT assay (details in Supporting Information), compounds 2, 4, 6, and 9 were found to be less cytotoxic at the concentrations utilized when compared with 10\% DMSO (Figure S1).

2.3. Impact of IRIs on the Post-Thaw Viability of UCB Committed and Multipotent Progenitors. The success of HSPC transplantation is directly correlated with both the number and quality of cells transplanted. ${ }^{24-31}$ The only test capable of identifying the presence of functional hematopoietic stem cells is the in vivo transplantation assay. ${ }^{33,34}$ This assay measures the ability of transplanted HSPCs (or LCs) to reconstitute the entire blood-forming system of an ablated host. $^{33,34}$ In this assay, hematopoietic reconstitution is measured in the peripheral blood samples at $\geq 4$ months post-transplantation. ${ }^{33,34}$ This time-consuming and expensive assay is not ideal for predicting the engraftment of banked CBUs. Therefore, transplant centers typically select HLAmatched units based on the TNC count, the number of CD34+ cells, and the number of committed progenitors detected using the CFU assay in the banked units. ${ }^{32}$ Importantly, patients who fail to engraft will often engraft successfully when another unit is given, indicating that conventional methods of graft selection are imperfect and do not discriminate units that will be unlikely to engraft. ${ }^{32} \mathrm{Up}$ to $20 \%$ of the patients receiving a UCB transplant will fail to fully engraft, in part because of inadequate potency of the unit. ${ }^{35-38}$

A recent study correlated prefreeze and post-thaw cell characteristics to determine the best indicators for successful engraftment and concluded that the CFU (committed progenitor) dose was the best indicator for successful engraftment. ${ }^{32}$ Typical post-thaw recoveries of TNCs of a cryopreserved cord unit were $>80 \%$, but the yield of colonies obtained in CFU assays after thawing was only $21.2 \%{ }^{32}$ On the basis of this measure of graft adequacy, only $2.8 \%$ of the inventory at a large cord blood bank would provide an adequate dose to ensure timely engraftment for a patient $>50 \mathrm{~kg} .{ }^{32} \mathrm{To}$ overcome this significant limitation, two or three separate UCB units are often transplanted in adult recipients to obtain a sufficient dose. ${ }^{39}$ This is, however, very costly and can lead to increased incidence of GVHD. The reduction in post-thaw CFU yields may reflect the damage to the unit during cryopreservation, shipping, and thawing. Therefore, the optimization of cryopreservation protocols for HSPCs, with an emphasis on the preservation of clonogenic CFU potential, is an important issue for optimizing storage in UCB banks.

Given that the CFU assay is one of the best indicators of engraftment potential, ${ }^{32,40}$ we sought to determine whether IRIs could improve the post-thaw recovery of committed progenitors of cryopreserved LCs. IRIs 2, 4, 6, and 9 were selected because of their high IRI activity and their low cytotoxicity levels (Figure S1). First, we tested whether the addition of IRIs 2, 4, 6, and 9 at concentrations lower than those used to assess IRI activity could improve the post-thaw recovery of committed progenitors. The increase in the number of committed progenitors in thawed LCs was observed for most committed progenitors, but the differences versus control (no compound) were not significant (Figure 4).

A concentration scan was performed to identify the optimal concentration for each IRI. Remarkably, significant increase ( $p$ 


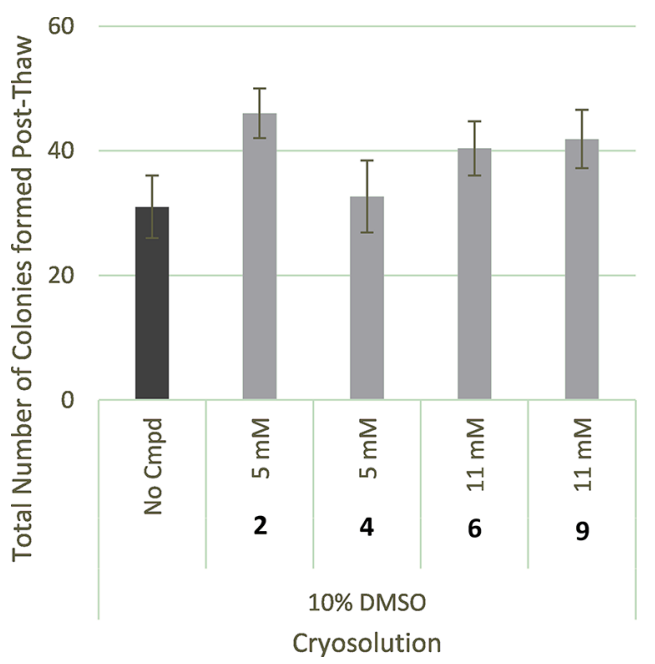

Figure 4. Total number of colonies formed (per $80 \mu \mathrm{L}$ thawed LC) post-thaw after cryopreservation of LCs in 10\% DMSO supplemented with IRI active molecules. Mean \pm SEM presented of 6-10 independent experiments. There is no statistical significance $(p>$ $0.05)$ as assessed using ANOVA with a Tukey multiple comparison test.

$<0.001,0.01$, or 0.05$)$ in the number of progenitors in a dosedependent manner was obtained when the standard cryoprotectant solution was supplemented with IRI active molecules (Figure 5). Compound 2 with the highest IRI activity was most effective at 12.5 and $25 \mathrm{mM}(p<0.05$ and 0.01$)$, producing 2fold increases in the number of committed progenitor cells when compared with the control. Compound 4 with a moderate IRI activity did not produce significantly more colonies than the control $(p>0.05)$. Compound 6 with an IRI activity equivalent to compound 2 produced the most colonies out of the compounds tested at $27.5 \mathrm{mM}(p<0.05)$, more than 2.4 times greater than the $10 \%$ DMSO cryoprotectant solution. Finally, compound 9 with the third highest IRI activity promoted progenitor recovery slightly lower than compounds 2 and 6.
There was no significant increase in the post-thaw viability of CD34+ cells when compared with the standard 10\% DMSO cryoprotectant solution in each case (Figure S2). In fact, the percentages of post-thaw CD34+ cell viability were poorly correlated with colony formation $\left(R^{2}=0.20\right.$, data not shown). These results further suggest that the post-thaw viability is not an accurate measure of cryopreservation success and UCB potency. However, although there were no differences in the percentages of post-thaw CD34+ cell viability, the net recovery of viable CD34+ cells was significantly greater with IRI 2 at 25 $\mathrm{mM}$ with a 1.6-fold increase when compared with the control $(p<0.0001)$ and IRI 9 at 18 and $55 \mathrm{mM}$, with a 1.4-fold increase when compared with the control $(p<0.05$ and 0.01 , Figure S3).

The number of CFU-GMs has been reported to be associated with the transplantation outcome of HSPCs from autologous $\mathrm{BM}$ and $\mathrm{UCB} .^{40-45}$ Furthermore, it has been reported that successfully transplanted UCB units had a higher number of CFU-GMs than those that were not transplanted. ${ }^{46}$ LCs supplemented with 6 or 9 resulted in a significant increase $(p<0.0001$ and 0.05$)$ in CFU-GEMM formation post-thaw (Table 1). Furthermore, IRI 6 increased the post-thaw yield of CFU-GM colonies (Table 1).

IRIs 2 and 6 were the most effective inhibitors of ice recrystallization and supported the largest increase in the number of post-thaw committed progenitors and CD34+ cell numbers, whereas the least IRI active compound (4) produced a similar number of progenitors as the DMSO control. To investigate whether this protective effect correlated with the ability to control ice growth and recrystallization, LCs were cryopreserved with structurally similar compounds $(3,5,7$, and 10) that did not exhibit an IRI activity. As expected, these compounds had very little effect on the CD34+ post-thaw viability (Figure S4A) and the total number of colonies formed in the CFU assay (Figure S4B). These results suggest that the compounds capable of controlling ice and recrystallization have a protective effect on the ability of UCB CD34+ cells and committed progenitors to proliferate and differentiate postthaw.
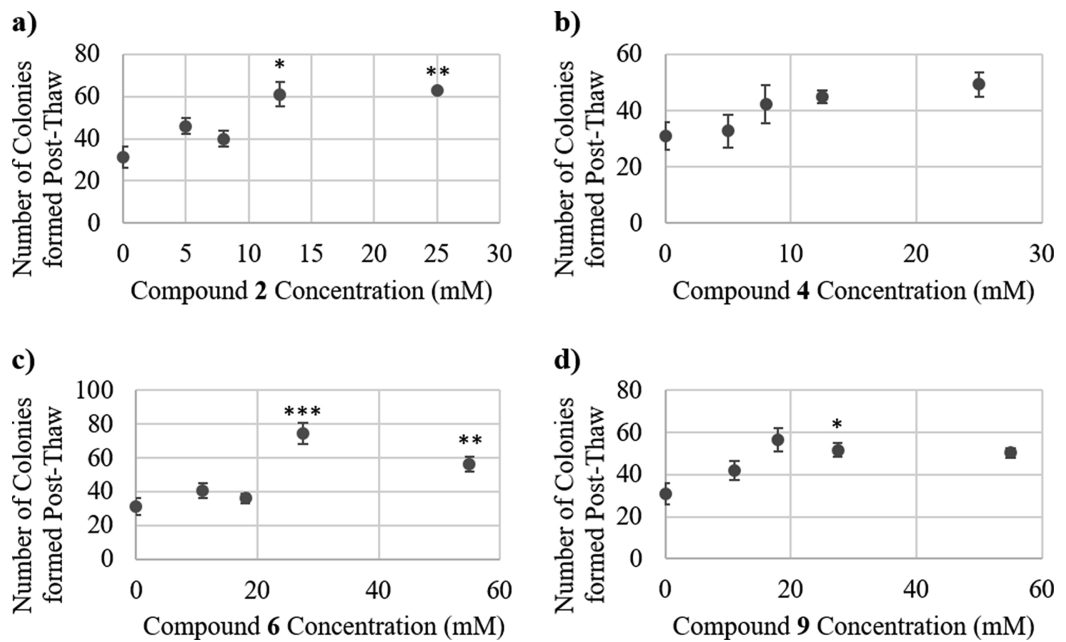

Figure 5. Dose response for the total number of colonies formed (per $80 \mu \mathrm{L}$ thawed LC) post-thaw after cryopreservation of LCs with $10 \%$ DMSO supplemented with various concentrations of (A) compound 2, (B) compound 4, (C) compound 6, or (D) compound 9. Mean \pm SEM presented of 2-10 independent experiments. Statistical significance, marked by asterisks, was assessed using ANOVA with a Dunnett's test for comparison to the control (no compound) with a $95 \%(*), 99 \%(* *)$, or $99.9 \%(* * *)$ confidence level. 
Table 1. Number of CFU-GM and CFU-GEMM Formed Post-Thaw and Proportion (Mean $\% \pm$ SD) of Viable, Necrotic, and Apoptotic CD34+ Cells Measured in LCs Post-Thaw

\begin{tabular}{|c|c|c|c|c|c|}
\hline cryoprotectant solution & $\mathrm{CFU}_{-G M s}{ }^{a}$ & CFU-GEMMs $^{a}$ & $\%$ viable $\mathrm{CD} 34+^{b}$ & $\%$ necrotic $\mathrm{CD} 34+^{b}$ & $\%$ apoptotic $\mathrm{CD} 34+^{b}$ \\
\hline 10\% DMSO (no cmpd) & $7( \pm 1.5)$ & $3( \pm 1.0)$ & $65.0( \pm 5.2)$ & $12.2( \pm 10.3)$ & $22.9( \pm 5.1)$ \\
\hline $10 \%$ DMSO + $2(12.5 \mathrm{mM})$ & $9( \pm 2.5)$ & $0( \pm 0.0)$ & $70.3( \pm 12.0)$ & $9.2( \pm 9.6)$ & $20.5( \pm 2.3)$ \\
\hline $10 \%$ DMSO + $2(25 \mathrm{mM})$ & $11( \pm 1.1)$ & $2( \pm 0.7)$ & $69.5( \pm 10.6)$ & $13.0( \pm 4.0)$ & $17.6( \pm 14.6)$ \\
\hline $10 \%$ DMSO $+6(27.5 \mathrm{mM})$ & $14( \pm 0.7)^{c}$ & $16( \pm 2.5)^{c}$ & $67.3( \pm 13.2)$ & $14.0( \pm 9.2)$ & $18.7( \pm 4.0)$ \\
\hline $10 \%$ DMSO + $9(18 \mathrm{mM})$ & $11( \pm 0.7)$ & $8( \pm 0.4)^{c}$ & $70.7( \pm 7.5)$ & $10.0( \pm 9.6)$ & $19.3( \pm 2.1)$ \\
\hline
\end{tabular}

${ }^{a}$ Mean \pm SEM presented of 4-10 independent experiments. The number of CFU-GM/GEMM (per $80 \mu \mathrm{L}$ thawed LC) formed post-thaw. ${ }^{b}$ Mean \pm SD presented of two independent experiments. Proportion of viable (Sytox- Annexin-), necrotic (Sytox+ Annexin+), and apoptotic (SytoxAnnexin $\mathrm{V}+$ ) CD34+ cells presented. 'Significant difference $(p<0.05)$ compared with $10 \%$ DMSO (no compound) as assessed using Student's $t$ test.
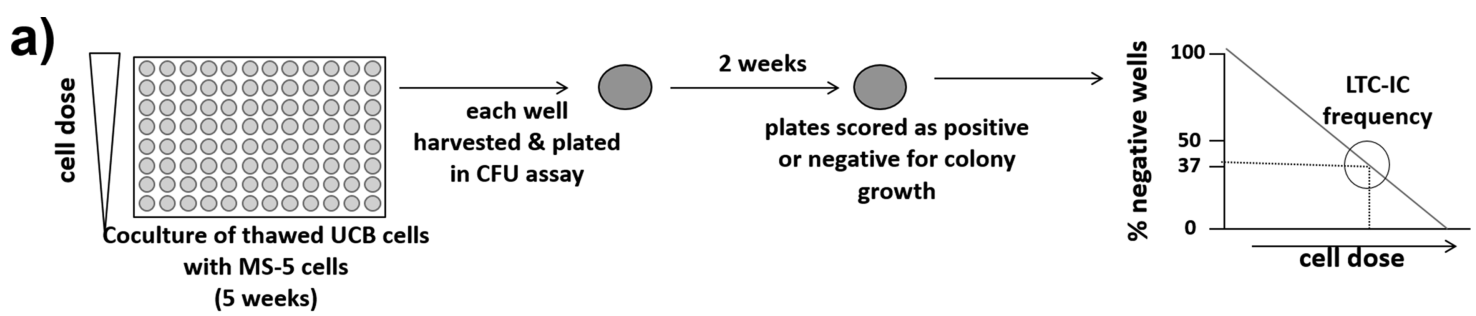

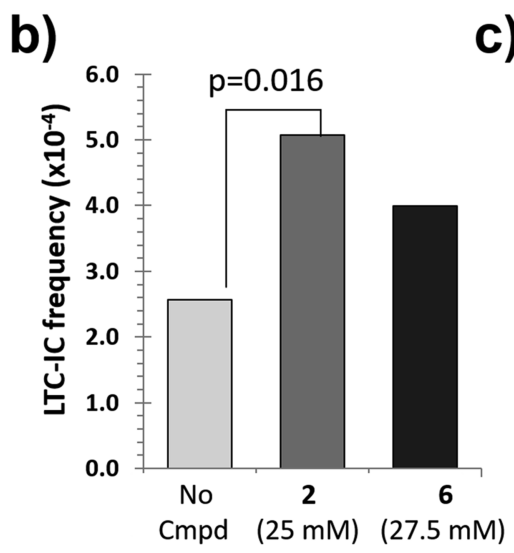

$10 \%$ DMSO c)

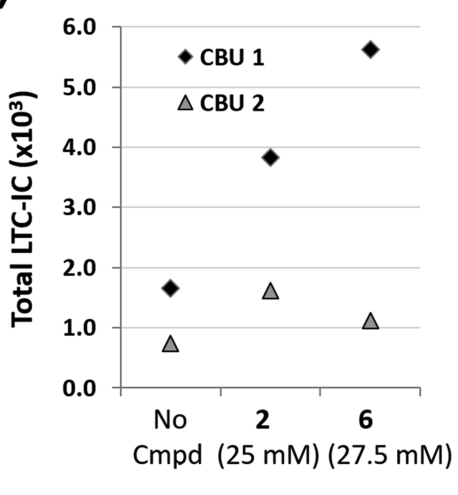

$10 \%$ DMSO d)

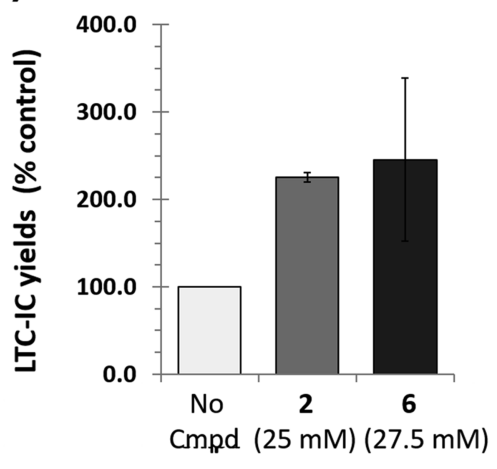

$10 \%$ DMSO

Figure 6. Frequency and the total number of LTC-ICs in post-thaw LCs after cryopreservation with $10 \%$ DMSO supplemented with IRIs 2 (25 $\mathrm{mM}$ ) and 6 (27.5 mM). (A) Overview of LDA used to measure the frequency of LTC-ICs in LCs. (B) Mean frequency of LTC-ICs estimated using ELDA $(n=2)$. (C) Total number of LTC-ICs obtained per thawed UCB vial for two units tested. The total number of LTC-ICs for each experiment was obtained by multiplying the measured TNC count with the frequency of LTC-ICs for each individual treatment. Each unit is indicated by different symbols. (D) Mean \pm SEM of the yield of LTC-ICs recovered $(n=2)$. Total number of LTC-ICs was normalized in each experiment to the total number obtained in the DMSO control [i.e., LTC-IC yield $=($ total LTC-IC with sample $) /($ total LTC-IC with no compound control $) \times 100 \%$ ].

The increased recovery of CFU indicated that IRIs 2, 6, and 9 improve the post-thaw functional ability of progenitors. To further investigate this cryoprotective effect of IRIs on more immature progenitors, we measured the frequency of multipotent progenitors in UCB LCs supplemented with the most effective IRIs $(2,6)$ using the stringent long-term cultureinitiating cell (LTC-IC) assay. This assay measures the frequency of progenitors with self-renewal and differentiation activities significantly superior to progenitors detected using the CFU assay. ${ }^{47}$ The frequency of LTC-IC in supplemented LCs was measured using limiting dilution analysis (LDA, Figure 6A). The frequencies of LTC-IC were increased up to 2-fold in post-thaw LCs supplemented with IRIs $2(25 \mathrm{mM}, p<0.05)$ and $6(27.5 \mathrm{mM}, p>0.05$, Figure $6 \mathrm{~B})$. Moreover, the total number of LTC-ICs recovered was always greater in the cryopreserved samples containing IRIs (Figure 6C), resulting in a 2.25-fold increase in LTC-IC yields versus control (Figure 6D).
Interestingly, the most effective IRIs in this study, $\mathbf{2}$ and $\mathbf{6}$, provided the greatest yield of committed progenitors and multipotent progenitors post-thaw. Moreover, the cryoprotective ability of the IRIs $\mathbf{2}$ and $\mathbf{6}$ was extended to more immature progenitors. Indeed, the frequency of multipotent progenitors in UCB LCs was significantly increased 2.5- and 3.2-fold over $10 \%$ DMSO. Moreover, the total yield of multipotent progenitors was increased 2 -fold over control. Importantly, the preservation of progenitor function during cryopreservation was confirmed to be a result of the molecules' ability to inhibit ice recrystallization. Structurally similar compounds devoid of IRI activity failed to improve the recovery of myeloid progenitors. Taken together, these results demonstrate that IRI activity during freezing and thawing of UCB LCs protects both committed and immature progenitors against cryo-injury that lead to a significant loss in UCB potency.

Finally, we investigated the impact of IRIs 2, 6, and 9 on CD34+ cell integrity and viability post-thaw using Annexin V 


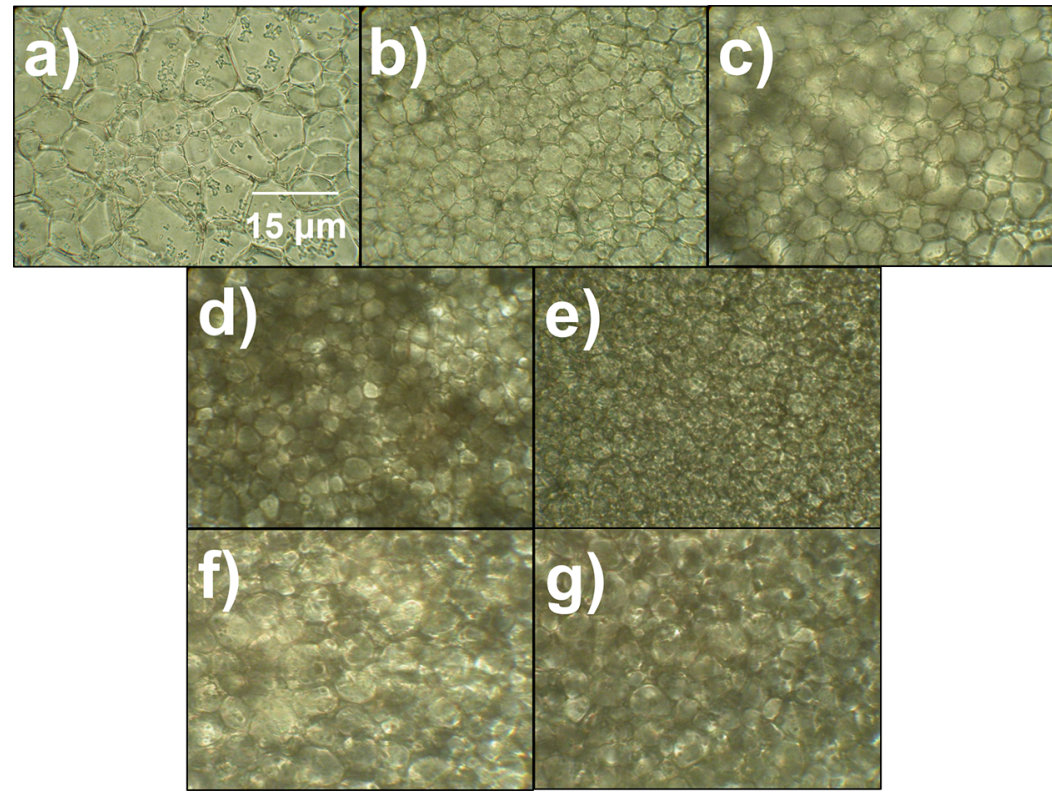

Figure 7. Images of ice crystals when LCs are in the presence of IRI active compounds after 30 min of ice recrystallization (at $\left.-6.4{ }^{\circ} \mathrm{C}\right)$. (A) PBS positive control for ice recrystallization. (B) Blood plasma. (C) LCs diluted with saline/dextran. (D) LCs in saline/dextran supplemented with 2 (25 $\mathrm{mM})$. (E) LCs in saline/dextran supplemented with 6 (27.5 mM). (F) LCs in saline/dextran supplemented with 3 (25 mM). (G) LCs in saline/ dextran supplemented with $7(55 \mathrm{mM})$.

staining, which detects apoptotic cells. Although no significant differences were identified, the proportion of viable CD34+ cells tended to be higher for all IRI-cryoprotected samples tested when compared with the DMSO control. The results suggest that this may be the result of decreased loss in CD34+ cells to apoptotis and/or necrosis, although these results will need to be confirmed and assessed further in future work. This could be significant because several studies have indicated that CD34+ cells can exhibit high levels of apoptosis post-thaw and as a result significant cell loss is experienced post-thaw. ${ }^{18,48-50}$ Furthermore, it has been shown that UCB CD34+ cells with signs of apoptosis (Annexin $\mathrm{V}+$ ) fail to engraft when transplanted into immunodeficient mice. ${ }^{18}$

2.4. Ice Recrystallization Inhibition Activity of $\mathbf{N}$-Arylaldonamides in the Presence of UCB Cells. The IRI activity of compounds $\mathbf{1 - 1 0}$ was measured utilizing PBS solutions containing $22 \mathrm{mM}$ of compound. Although this is our standard method for measuring the IRI activity of newly synthesized molecules, the cryoprotectant solutions were composed of IRI dissolved in $0.9 \%$ saline $/ 0.5 \%$ dextran (from Leuconostoc spp., $M_{\mathrm{r}} \approx 40000$, Sigma) in water at concentrations different from $22 \mathrm{mM}$ and in the presence of plasma and 10\% DMSO. When assessed in PBS, compounds 2 and 6 were strong IRIs, whereas compounds 3 and 7 were inactive (Figure 2). We predicted that IRI active compounds would retain their IRI activity in the presence of LCs and ice crystals should be noticeably smaller in size in the presence of an active IRI. Thus, solutions of LCs with active and inactive IRI compounds were assessed for ice recrystallization inhibition activity (Figure 7). The 10\% DMSO was excluded from the solutions because, in practice, concentrations above $6 \%(\mathrm{v} / \mathrm{v})$ are very difficult to use in this assay and produce inconsistent results because of large portions of unfrozen solution in the ice wafer. ${ }^{51}$ Compared with PBS (Figure 7, panel A), blood plasma alone possesses remarkable IRI activity, with a MGS of $13 \%$ (Figure 7, panel B). This is not surprising as plasma contains many different lipids, carbohydrates, proteins, and salts. LCs with saline/dextran (cryoprotectant solution without DMSO or IRI) are less active (24\% MGS) when compared with plasma alone that can be attributed to the dilution of plasma with saline/dextran (Figure 7, panel C). LCs in plasma and saline/ dextran supplemented with compounds $2(25 \mathrm{mM})$ and 6 (27.5 $\mathrm{mM}$ ) show increased IRI activity when compared with LCs in plasma and saline/dextran alone with activities of 9 and 3\%, respectively (Figure 7, panels D and E). By contrast, ineffective IRIs $3(25 \mathrm{mM})$ and $7(55 \mathrm{mM})$ that are structurally similar to 2 and 6, respectively, had IRI activities of 18 and 17\%, respectively (Figure 7, panels $\mathrm{F}$ and $\mathrm{G}$ ). These results obtained in LCs confirm the previous results of IRI activity and support the concept that IRI active compounds $\mathbf{2}$ and $\mathbf{6}$ are potent inhibitors of ice recrystallization in UCB cells during cryopreservation.

In summary, this study identified a series of small molecules capable of controlling ice growth and recrystallization. Compounds $\mathbf{2}$ and $\mathbf{6}$ with the most potent IRI activity significantly improved the post-thaw recovery of committed progenitors and multipotent progenitors when supplemented with the standard cryoprotectant solution in LCs. These results together with the strong correlation between engraftment and net TNC and progenitor content (i.e., dose) reported in clinical studies support the hypothesis that the use of IRIs during cryopreservation could improve the engraftment capacity of UCB. Future work will aim to test this hypothesis by performing the transplantation of IRI-cryopreserved UCB cells into immunodeficient mice. Moreover, the capacity of these IRIs to protect other cellular products such as mesenchymal stromal cells will also need to be investigated. In conclusion, this study demonstrates that the inhibition of ice recrystallization during freezing and thawing through the addition of IRIs significantly improves the post-thaw potency of the cryopreserved UCB. 


\section{EXPERIMENTAL SECTION}

3.1. Splat-Cooling Assay for Ice Recrystallization Inhibition Activity. The assessment of IRI activity has been previously described. Briefly, IRI activity is determined using a method termed "splat-cooling" assay. ${ }^{52}$ In this method, an analyte is dissolved in PBS, and a $10 \mu \mathrm{L}$ droplet of this solution is dropped from a micropipette through a $2 \mathrm{~m}$ high plastic tube (10 cm in diameter) onto a block of polished aluminum precooled to approximately $-80{ }^{\circ} \mathrm{C}$. The droplet freezes instantly on the polished aluminum block. The resulting wafer is carefully removed from the surface of the block and transferred to a cryostage held at $-6.4{ }^{\circ} \mathrm{C}$ for annealing. After a period of $30 \mathrm{~min}$, the wafer is photographed between crossed polarizing filters using a digital camera (Nikon CoolPix 5000) fitted to the microscope. A total of three images are taken from each wafer, and this process is repeated with two additional wafers. During flash freezing, ice crystals spontaneously nucleate from the supercooled solution. These initial crystals are relatively homogeneous in size and quite small. During the annealing cycle, recrystallization occurs, resulting in a dramatic increase in the ice crystal size. Image analysis of the ice wafers was performed using domain recognition software. IRI activity is reported as a percentage of MGS of ice crystals in the presence of the solute when compared with the MGS of ice crystals in a control solution of PBS.

3.2. Collection and Processing of Human Umbilical Cord Blood. UCB was collected following healthy term delivery and informed consent from mothers, in accordance with institutional approval from the Research Ethics Board of The Ottawa Hospital (protocol 2006460-01H). The UCB was processed within $48 \mathrm{~h}$ of collection. The UCB was diluted with $6 \%$ hetastarch (Hespan) to obtain a final concentration of $1 \%$ hetastarch and incubated for $10 \mathrm{~min}$ at room temperature. ${ }^{8}$ The tubes were then centrifuged at $50 \mathrm{~g}$ at $10^{\circ} \mathrm{C}$ for $15-20 \mathrm{~min}$. For total volumes up to $30 \mathrm{~mL}, 15-17 \mathrm{~min}$ centrifugation was carried out, and for total volumes of $30-35 \mathrm{~mL}, 17-20 \mathrm{~min}$ centrifugation was carried out. The supernatant and buffy coat (plasma and leukocytes) were removed carefully (not collecting RBCs) from the tubes and were collected in a $50 \mathrm{~mL}$ Falcon tube. This tube was centrifuged at $400 \mathrm{~g}$ and $10{ }^{\circ} \mathrm{C}$ for $10 \mathrm{~min}$ to pellet cells. The plasma was removed and kept on ice. The cell pellet was resuspended and combined in plasma to a total volume of $20 \mathrm{~mL}$ (LC). The LC was kept on ice for the duration of its use. Total mononuclear (CD45+) and CD34+ cell concentrations and viability were determined using flow cytometry.

3.3. Cryopreservation of CD34+ Cells. Aliquots of LC containing $50000 \mathrm{CD} 34+$ cells were added to $2.0 \mathrm{~mL}$ cryovials. Cryovials were centrifuged at $400 \mathrm{~g}$ and $10{ }^{\circ} \mathrm{C}$ for $5 \mathrm{~min}$, the supernatant was removed, the plasma was added, and the cells were suspended by pipetting. Pre-made cryoprotectant solutions were added, and the cells were mixed by pipetting. For specific volumes of plasma and cryoprotectant solutions, see the Supporting Information. Cryovials were placed in a Mr. Frosty rate-controlled freezing container, which was then placed in a $-80{ }^{\circ} \mathrm{C}$ freezer for $24 \mathrm{~h}$. After $24 \mathrm{~h}$, the cryovials were transferred to a liquid nitrogen Dewar flask for storage until thawed for analysis.

3.4. Post-Thaw Recovery and Viability of CD34+ and CD45+ Cells from LCs. Flow cytometry analysis was performed using the ISHAGE-gating strategy (International Society of Hematotherapy and Graft Engineering) for CD45+ and CD34+ cell measurements. ${ }^{53}$ Frozen LC samples were thawed in a $37{ }^{\circ} \mathrm{C}$ water bath and analyzed without washing. In short, the LC was diluted with 10-fold Dulbecco's phosphate buffered saline (DPBS), $200 \mu \mathrm{L}$ was stained with CD45 fluorescein isothiocyanate (FITC) and CD34 phycoerythrin (PE) antibodies, and the mixture was incubated in the dark at room temperature. 7-AAD was added and incubated for 5 min. Finally, $20 \mu \mathrm{L}$ of Countbright counting beads (Life Technologies) was added, and the suspension was diluted to $1 \mathrm{~mL}$ with $1 \times \mathrm{RBC}$ lysis buffer (BioLegend). Samples were analyzed using a Beckman Coulter Gallios flow cytometer. The proportions of viable, apoptotic, and necrotic cells were measured by staining LC with CD34-PE, CD45RA-allophycocyanin (APC), Annexin V-FITC, and Sytox following the manufacturer's directions (Life Technologies).

3.5. CFU and Long-Term Culture-Initiating Cell (LTCIC) Assays. Cryovials were thawed in a $37{ }^{\circ} \mathrm{C}$ water bath and diluted with $900 \mu \mathrm{L}$ of Iscove's modified Dulbecco's media (IMDM) (10\% fetal bovine serum (FBS), 1\% penicillin/ streptomycin). Cryovials were placed on ice until washed. The samples were mixed by pipetting, and $80 \mu \mathrm{L}$ was transferred to a $15 \mathrm{~mL}$ Falcon tube. IMDM (10\% FBS; $5 \mathrm{~mL})$ was added, and this mixture was centrifuged at $1100 \mathrm{rpm}$ for $6 \mathrm{~min}$. The supernatant was removed by aspiration, and the cell pellet was resuspended in $1 \mathrm{~mL}$ of IMDM ( $2 \%$ FBS). The suspension was mixed by pipetting. $150 \mu \mathrm{L}$ of this cell suspension was added to $3 \mathrm{~mL}$ of Methocult media, and plating was performed as previously described. ${ }^{54}$ Colonies were counted and scored at day 14 according to the Standardized Guide provided by manufacturer. LDA was used to measure the frequency of LTCIC in thawed cryovials by plating decreasing doses of CB. TNCs $(250,600,1200,3000,6000,9000,18000$, and 30000$)$ were co-cultured for 5 weeks with the stromal cell line MS-5 on $0.1 \%$ gelatin precoated 96 -well flat bottom culture plates in a human long-term culture medium (Myelocult MS 300) with hydrocortisone. Half medium change was carried out weekly. After 5 weeks, the entire well content was transferred to $1 \mathrm{~mL}$ of methylcellulose (MethoCult H4434), and the plates were scored for colony growth or not after 2 weeks. Each dose was tested in 4-9 replicates. LDA was carried out using the extreme limiting dilution analysis (ELDA) web-based application. ${ }^{55}$ All reagents for progenitor assays were from StemCell Technologies.

\section{ASSOCIATED CONTENT}

S Supporting Information

The Supporting Information is available free of charge on the ACS Publications website at DOI: 10.1021/acsomega.6b00178.

Detailed description of all methods (PDF)

\section{AUTHOR INFORMATION}

\section{Corresponding Author}

*E-mail: rben@uottawa.ca. Phone: (613) 562-5800 (R.N.B.).

Notes

The authors declare no competing financial interest.

\section{ACKNOWLEDGMENTS}

The authors acknowledge the Natural Sciences and Engineering Research Council of Canada (NSERC), the Canadian Institutes of Health Research (CIHR), the Canadian Blood Services (CBS), and the Canada Research Chairs Program for financial support. The views expressed herein do not necessarily 
represent the views of the federal government. J.G.B. thanks CBS for a GFP award.

\section{REFERENCES}

(1) Hunt, C. J. Cryopreservation of Human Stem Cells for Clinical Application: A Review. Transfus. Med. Hemother. 2011, 38, 107-123.

(2) Harris, D. T. Non-Haematological Uses of Cord Blood Stem Cells. Br. J. Haematol. 2009, 147, 177-184.

(3) Iafolla, M. A. J.; Tay, J.; Allan, D. S. Transplantation of Umbilical Cord Blood-Derived Cells for Novel Indications in Regenerative Therapy or Immune Modulation: A Scoping Review of Clinical Studies. Biol. Blood Marrow Transplant. 2014, 20, 20-25.

(4) Yoder, M. C. Cord Blood Banking and Transplantation: Advances and Controversies. Curr. Opin. Pediatr. 2014, 26, 163-168.

(5) Bender, J. G.; Unverzagt, K.; Walker, D. E.; Lee, W.; Smith, S.; Williams, S.; Van Epps, D. E. Phenotypic Analysis and Characterization of CD34+ Cells from Normal Human Bone Marrow, Cord Blood, Peripheral Blood, and Mobilized Peripheral Blood from Patients Undergoing Autologous Stem Cell Transplantation. Clin. Immunol. Immunopathol. 1994, 70, 10-18.

(6) Gluckman, E. Ten Years of Cord Blood Transplantation: From Bench to Bedside. Br. J. Haematol. 2009, 147, 192-199.

(7) Berz, D.; McCormack, E. M.; Winer, E. S.; Colvin, G. A.; Quesenberry, P. J. Cryopreservation of Hematopoietic Stem Cells. Am. J. Hematol. 2007, 82, 463-472.

(8) Rubinstein, P.; Dobrila, L.; Rosenfield, R. E.; Adamson, J. W.; Migliaccio, G.; Migliaccio, A. R.; Taylor, P. E.; Stevens, C. E. Processing and Cryopreservation of Placental/Umbilical Cord Blood for Unrelated Bone Marrow Reconstitution. Proc. Natl. Acad. Sci. U.S.A. 1995, 92, 10119-10122.

(9) Davis, J.; Rowley, S. D.; Santos, G. W. Toxicity of Autologous Bone Marrow Graft Infusion. Prog. Clin. Biol. Res. 1990, 333, 531-540.

(10) Davis, J. M.; Rowley, S. D.; Braine, H. G.; Piantadosi, S.; Santos, G. W. Clinical Toxicity of Cryopreserved Bone Marrow Graft Infusion. Blood 1990, 75, 781-786.

(11) Alessandrino, E. P.; Bernasconi, P.; Caldera, D.; Colombo, A.; Bonfichi, M.; Malcovati, L.; Klersy, C.; Martinelli, G.; Maiocchi, M.; Pagnucco, G.; Varettoni, M.; Perotti, C.; Bernasconi, C. Adverse Events Occurring during Bone Marrow or Peripheral Blood Progenitor Cell Infusion: Analysis of 126 Cases. Bone Marrow Transplant. 1999, 23, 533-537.

(12) Zambelli, A.; Poggi, G.; Da Prada, G.; Pedrazzoli, P.; Cuomo, A.; Miotti, D.; Perotti, C.; Preti, P.; della Cuna, G. R. Clinical Toxicity of Cryopreserved Circulating Progenitor Cells Infusion. Anticancer Res. 1998, 18, 4705-4708.

(13) Woods, E. J.; Liu, J.; Derrow, C. W.; Smith, F. O.; Williams, D. A.; Critser, J. K. Cutting Edge Communication: Osmometric and Permeability Characteristics of Human Placental/Umbilical Cord Blood CD34+ Cells and Their Application to Cryopreservation. J. Hematother. Stem Cell Res. 2000, 9, 161-173.

(14) Halle, P.; Tournilhac, O.; Knopinska-Posluszny, W.; Kanold, J.; Gembara, P.; Boiret, N.; Rapatel, C.; Berger, M.; Travade, P.; Angielski, S.; Bonhomme, J.; Deméocq, F. Uncontrolled-Rate Freezing and Storage at $-80{ }^{\circ} \mathrm{C}$, with Only 3.5-Percent DMSO in Cryoprotective Solution for 109 Autologous Peripheral Blood Progenitor Cell Transplantations. Transfusion 2001, 41, 667-673.

(15) Beaujean, F.; Bourhis, J.-H.; Bayle, C.; Jouault, H.; Divine, M.; Rieux, C.; Janvier, M.; Le Forestier, C.; Pico, J. L. Successful Cryopreservation of Purified Autologous CD34+ Cells: Influence of Freezing Parameters on Cell Recovery and Engraftment. Bone Marrow Transplant. 1998, 22, 1091.

(16) Yang, H.; Acker, J. P.; Cabuhat, M.; Letcher, B.; Larratt, L.; McGann, L. E. Association of Post-Thaw Viable CD34+ Cells and CFU-GM with Time to Hematopoietic Engraftment. Bone Marrow Transplant. 2005, 35, 881-887.

(17) Galmes, A.; Gutiérrez, A.; Sampol, A.; Canaro, M.; Morey, M.; Iglesias, J.; Matamoros, N.; Duran, M. A.; Novo, A.; Bea, M. D.; Galán, P.; Balansat, J.; Martínez, J.; Bargay, J.; Besalduch, J. Long-Term Hematologic Reconstitution and Clinical Evaluation of Autologous
Peripheral Blood Stem Cell Transplantation after Cryopreservation of Cells with $5 \%$ and $10 \%$ Dimethylsulfoxide at $-80{ }^{\circ} \mathrm{C}$ in a Mechanical Freezer. Haematologica 2007, 92, 986-989.

(18) Shim, J.-S.; Cho, B.; Kim, M.; Park, G.-S.; Shin, J.-C.; Hwang, H.-K.; Kim, T.-G.; Oh, I.-H. Early Apoptosis in CD34 ${ }^{+}$Cells as a Potential Heterogeneity in Quality of Cryopreserved Umbilical Cord Blood. Br. J. Haematol. 2006, 135, 210-213.

(19) Wu, L.; Al-Hejazi, A.; Filion, L.; Ben, R.; Halpenny, M.; Yang, L.; Giulivi, A.; Allan, D. S. Increased Apoptosis in Cryopreserved Autologous Hematopoietic Progenitor Cells Collected by Apheresis and Delayed Neutrophil Recovery after Transplantation: A Nested Case-Control Study. Cytotherapy 2012, 14, 205-214.

(20) Sasnoor, L. M.; Kale, V. P.; Limaye, L. S. Supplementation of Conventional Freezing Medium with a Combination of Catalase and Trehalose Results in Better Protection of Surface Molecules and Functionality of Hematopoietic Cells. J. Hematother. Stem Cell Res. 2003, 12, 553-564.

(21) Sasnoor, L. M.; Kale, V. P.; Limaye, L. S. A Combination of Catalase and Trehalose as Additives to Conventional Freezing Medium Results in Improved Cryoprotection of Human Hematopoietic Cells with Reference to in Vitro Migration and Adhesion Properties. Transfusion 2005, 45, 622-633.

(22) Sasnoor, L. M.; Kale, V. P.; Limaye, L. S. Prevention of Apoptosis as a Possible Mechanism behind Improved Cryoprotection of Hematopoietic Cells by Catalase and Trehalose. Transplantation 2005, 80, 1251-1260.

(23) Wu, L. K.; Tokarew, J. M.; Chaytor, J. L.; von Moos, E.; Li, Y.; Palii, C.; Ben, R. N.; Allan, D. S. Carbohydrate-Mediated Inhibition of Ice Recrystallization in Cryopreserved Human Umbilical Cord Blood. Carbohydr. Res. 2011, 346, 86-93.

(24) Allan, D. S.; Keeney, M.; Howson-Jan, K.; Popma, J.; Weir, K.; Bhatia, M.; Sutherland, D. R.; Chin-Yee, I. H. Number of Viable $\mathrm{CD}^{+} 4^{+}$Cells Reinfused Predicts Engraftment in Autologous Hematopoietic Stem Cell Transplantation. Bone Marrow Transplant. 2002, $29,967$.

(25) Abrahamsen, J. F.; Wentzel-Larsen, T.; Bruserud, Ø. Autologous Transplantation: The Viable Transplanted $\mathrm{CD}_{3} 4^{+}$Cell Dose Measured Post-Thaw Does Not Predict Engraftment Kinetics Better than the Total $\mathrm{CD} 34^{+}$Cell Dose Measured Pre-Freeze in Patients That Receive More than $2 \times 10^{6} \mathrm{CD} 34^{+}$Cells $/ \mathrm{kg}$. Cytotherapy 2004, 6, 356-362.

(26) Carral, A.; de la Rubia, J.; Martín, G.; Mollá, S.; Martínez, J.; Sanz, G. F.; Soler, M. A.; Jarque, I.; Jiménez, C.; Sanz, M. A. Factors Influencing the Collection of Peripheral Blood Stem Cells in Patients with Acute Myeloblastic Leukemia and Non-Myeloid Malignancies. Leuk. Res. 2003, 27, 5-12.

(27) Sieburg, H. B.; Cho, R. H.; Müller-Sieburg, C. E. Limiting Dilution Analysis for Estimating the Frequency of Hematopoietic Stem Cells: Uncertainty and Significance. Exp. Hematol. 2002, 30, $1436-1443$.

(28) Theilgaard-Mönch, K.; Raaschou-Jensen, K.; Heilmann, C.; Andersen, H.; Bock, J.; Russel, C. A.; Vindeløv, L.; Jacobsen, N.; Dickmeiss, E. A Comparative Study of $\mathrm{CD} 34^{+}$Cells, CD $34^{+}$Subsets, Colony Forming Cells and Cobblestone Area Forming Cells in Cord Blood and Bone Marrow Allografts. Eur. J. Haematol. 1999, 62, 174183.

(29) Zaucha, J. M.; Gooley, T.; Bensinger, W. I.; Heimfeld, S.; Chauncey, T. R.; Zaucha, R.; Martin, P. J.; Flowers, M. E. D.; Storek, J.; Georges, G.; Storb, R.; Torok-Storb, B. CD34 Cell Dose in Granulocyte Colony-Stimulating Factor-mobilized Peripheral Blood Mononuclear Cell Grafts Affects Engraftment Kinetics and Development of Extensive Chronic Graft-versus-Host Disease After Human Leukocyte Antigen-Identical Sibling Transplantation. Blood 2001, 98, 3221-3227.

(30) Donaldson, C.; Armitage, W. J.; Denning-Kendall, P. A.; Nicol, A. J.; Bradley, B. A.; Hows, J. M. Optimal Cryopreservation of Human Umbilical Cord Blood. Bone Marrow Transplant. 1996, 18, 725-731.

(31) Gao, D. Y.; Chang, Q.; Liu, C.; Farris, K.; Harvey, K.; McGann, L. E.; English, D.; Jansen, J.; Critser, J. K. Fundamental Cryobiology of 
Human Hematopoietic Progenitor Cells. I: Osmotic Characteristics and Volume Distribution. Cryobiology 1998, 36, 40-48.

(32) Page, K. M.; Zhang, L.; Mendizabal, A.; Wease, S.; Carter, S.; Gentry, T.; Balber, A. E.; Kurtzberg, J. Total Colony-Forming Units are a Strong, Independent Predictor of Neutrophil and Platelet Engraftment after Unrelated Umbilical Cord Blood Transplantation: A Single-Center Analysis of 435 Cord Blood Transplants. Biol. Blood Marrow Transplant. 2011, 17, 1362-1374.

(33) Purton, L. E.; Scadden, D. T. Limiting Factors in Murine Hematopoietic Stem Cell Assays. Cell Stem Cell 2007, 1, 263-270.

(34) Harrison, D. E. Competitive Repopulation: A New Assay for Long-Term Stem Cell Functional Capacity. Blood 1980, 55, 77-81.

(35) Kurtzberg, J.; Prasad, V. K.; Carter, S. L.; Wagner, J. E.; BaxterLowe, L. A.; Wall, D.; Kapoor, N.; Guinan, E. C.; Feig, S. A.; Wagner, E. L.; Kernan, N. A. Results of the Cord Blood Transplantation Study (COBLT): Clinical Outcomes of Unrelated Donor Umbilical Cord Blood Transplantation in Pediatric Patients with Hematologic Malignancies. Blood 2008, 112, 4318-4327.

(36) Martin, P. L.; Carter, S. L.; Kernan, N. A.; Sahdev, I.; Wall, D.; Pietryga, D.; Wagner, J. E.; Kurtzberg, J. Results of the Cord Blood Transplantation Study (COBLT): Outcomes of Unrelated Donor Umbilical Cord Blood Transplantation in Pediatric Patients with Lysosomal and Peroxisomal Storage Diseases. Biol. Blood Marrow Transplant. 2006, 12, 184-194.

(37) Eapen, M.; Rocha, V.; Sanz, G.; Scaradavou, A.; Zhang, M.-J.; Arcese, W.; Sirvent, A.; Champlin, R. E.; Chao, N.; Gee, A. P.; Isola, L.; Laughlin, M. J.; Marks, D. I.; Nabhan, S.; Ruggeri, A.; Soiffer, R.; Horowitz, M. M.; Gluckman, E.; Wagner, J. E. Effect of Graft Source on Unrelated Donor Haemopoietic Stem-Cell Transplantation in Adults with Acute Leukaemia: A Retrospective Analysis. Lancet Oncol. 2010, 11, 653-660.

(38) Barker, J. N.; Scaradavou, A.; Stevens, C. E. Combined Effect of Total Nucleated Cell Dose and HLA Match on Transplantation Outcome in 1061 Cord Blood Recipients with Hematologic Malignancies. Blood 2009, 115, 1843-1849.

(39) Jaing, T.-H.; Yang, C.-P.; Hung, I.-J.; Chen, S.-H.; Sun, C.-F.; Chow, R. Transplantation of Unrelated Donor Umbilical Cord Blood Utilizing Double-Unit Grafts for Five Teenagers with TransfusionDependent Thalassemia. Bone Marrow Transplant. 2007, 40, 307-311.

(40) Migliaccio, A. R.; Adamson, J. W.; Stevens, C. E.; Dobrila, N. L.; Carrier, C. M.; Rubinstein, P. Cell Dose and Speed of Engraftment in Placental/Umbilical Cord Blood Transplantation: Graft Progenitor Cell Content is a Better Predictor than Nucleated Cell Quantity. Blood 2000, 96, 2717-2722.

(41) Emminger, W.; Emminger-Schmidmeier, W.; Höcker, P.; Hinterberger, W.; Gadner, H. Myeloid Progenitor Cells (CFU-GM) Predict Engraftment Kinetics in Autologous Transplantation in Children. Bone Marrow Transplant. 1989, 4, 415-420.

(42) Al-Fiar, F.; Prince, H. M.; Imrie, K.; Stewart, A. K.; Crump, M.; Keating, A. Bone Marrow Mononuclear Cell Count Does Not Predict Neutrophil and Platelet Recovery Following Autologous Bone Marrow Transplant: Value of the Colony-Forming Unit Granulocyte-Macrophage (CFU-GM) Assay. Cell Transplant. 1997, 6, 491-495.

(43) Douay, L.; Gorin, N. C.; Mary, J. Y.; Lemarie, E.; Lopez, M.; Najman, A.; Stachowiak, J.; Giarratana, M. C.; Baillou, C.; Salmon, C.; et al. Recovery of CFU-GM from Cryopreserved Marrow and in Vivo Evaluation after Autologous Bone Marrow Transplantation are Predictive of Engraftment. Exp. Hematol. 1986, 14, 358-365.

(44) Lee, Y. H.; Cho, N. C.; Je, K. H.; Han, H.; Han, J. Y.; Kim, J. S.; Kim, H. J.; Cho, B.; Kim, H. K. Successful Sibling Cord Blood Stem Cell Transplantation for Relapsed Acute Mixed Lineage Leukemia. Korean J. Hematol. 1999, 34, 471-476.

(45) Prasad, V. K.; Mendizabal, A.; Parikh, S. H.; Szabolcs, P.; Driscoll, T. A.; Page, K.; Lakshminarayanan, S.; Allison, J.; Wood, S.; Semmel, D.; Escolar, M. L.; Martin, P. L.; Carter, S.; Kurtzberg, J. Unrelated Donor Umbilical Cord Blood Transplantation for Inherited Metabolic Disorders in 159 Pediatric Patients from a Single Center: Influence of Cellular Composition of the Graft on Transplantation Outcomes. Blood 2008, 112, 2979-2989.
(46) Yoo, K. H.; Lee, S. H.; Kim, H.-J.; Sung, K. W.; Jung, H. L.; Cho, E. J.; Park, H. K.; Kim, H. A.; Koo, H. H. The Impact of Post-Thaw Colony-Forming Units-Granulocyte/Macrophage on Engraftment Following Unrelated Cord Blood Transplantation in Pediatric Recipients. Bone Marrow Transplant. 2007, 39, 515-521.

(47) Coulombel, L. Identification of Hematopoietic Stem/Progenitor Cells: Strength and Drawbacks of Functional Assays. Oncogene 2004, $23,7210-7222$.

(48) de Boer, F.; Dräger, A. M.; Pinedo, H. M.; Kessler, F. L.; Monnee-van Muijen, M.; Weijers, G.; Westra, G.; van der Wall, E.; Netelenbos, T.; Oberink, J. W.; Huijgens, P. C.; Schuurhuis, G. J. Early Apoptosis Largely Accounts for Functional Impairment of CD34 ${ }^{+}$ Cells in Frozen-Thawed Stem Cell Grafts. J. Hematother. Stem Cell Res. 2002, 11, 951-963.

(49) de Boer, F.; Dräger, A. M.; Pinedo, H. M.; Kessler, F. L.; van der Wall, E.; Jonkhoff, A. R.; van der Lelie, J.; Huijgens, P. C.; Ossenkoppele, G. J.; Schuurhuis, G. J. Extensive Early Apoptosis in Frozen-Thawed CD34-Positive Stem Cells Decreases Threshold Doses for Haematological Recovery after Autologous Peripheral Blood Progenitor Cell Transplantation. Bone Marrow Transplant. 2002, 29, 249-255.

(50) Xiao, M.; Dooley, D. C. Assessment of Cell Viability and Apoptosis in Human Umbilical Cord Blood Following Storage. J. Hematother. Stem Cell Res. 2003, 12, 115-122.

(51) Tam, R. Y.; Ferreira, S. S.; Czechura, P.; Chaytor, J. L.; Ben, R. N. Hydration Index-A Better Parameter for Explaining Small Molecule Hydration in Inhibition of Ice Recrystallization. J. Am. Chem. Soc. 2008, 130, 17494-17501.

(52) Knight, C. A.; Hallett, J.; DeVries, A. L. Solute Effects on Ice Recrystallization: An Assessment Technique. Cryobiology 1988, 25, $55-60$.

(53) Sutherland, D. R.; Anderson, L.; Keeney, M.; Nayar, R.; ChinYee, I. The ISHAGE Guidelines for $\mathrm{CD} 34^{+}$Cell Determination by Flow Cytometry. J. Hematother. 1996, 5, 213-226.

(54) Petzer, A. L.; Hogge, D. E.; Landsdorp, P. M.; Reid, D. S.; Eaves, C. J. Self-Renewal of Primitive Human Hematopoietic Cells (LongTerm-Culture-Initiating Cells) in Vitro and Their Expansion in Defined Medium. Proc. Natl. Acad. Sci. U.S.A. 1996, 93, 1470-1474.

(55) Hu, Y.; Smyth, G. K. ELDA: Extreme Limiting Dilution Analysis for Comparing Depleted and Enriched Populations in Stem Cell and Other Assays. J. Immunol. Methods 2009, 347, 70-78. 